\title{
Towards a description of the Kondo effect using time-dependent density functional theory
}

\author{
G. Stefanucci ${ }^{1,2,3}$ and S. Kurth ${ }^{4,5,3}$ \\ ${ }^{1}$ Dipartimento di Fisica, Università di Roma Tor Vergata, \\ Via della Ricerca Scientifica 1, 00133 Rome, Italy \\ ${ }^{2}$ INFN, Laboratori Nazionali di Frascati, Via E. Fermi 40, 00044 Frascati, Italy \\ ${ }^{3}$ European Theoretical Spectroscopy Facility (ETSF) \\ ${ }^{4}$ Nano-Bio Spectroscopy Group, Dpto. de Física de Materiales, Universidad del País Vasco UPV/EHU, \\ Centro Física de Materiales CSIC-UPV/EHU, Av. Tolosa 72, E-20018 San Sebastián, Spain \\ ${ }^{5}$ IKERBASQUE, Basque Foundation for Science, E-48011 Bilbao, Spain
}

(Dated: October 1, 2018)

\begin{abstract}
We demonstrate that the zero-temperature conductance of the Anderson model can be calculated within the Landauer formalism combined with static density functional theory (DFT). The proposed approximate functional is based on finite-temperature DFT and yields the exact Kohn-Sham potential at the particle-hole symmetric point. Furthermore, in the limit of zero temperature it correctly exhibits a derivative discontinuity which is shown to be essential to reproduce the conductance plateau. On the other hand, at the Kondo temperature the exact Kohn-Sham conductance overestimates the real one by an order of magnitude. To understand the failure of DFT we resort to its time-dependent version and conclude that the suppression of the Kondo resonance must be attibuted to dynamical exchange-correlation corrections.
\end{abstract}

PACS numbers: 31.15.ee, 72.10.Fk, 05.60.Gg

Despite the many successes of density functional theory (DFT) [1] in the description of the electronic structure of many-electron systems, the treatment of strongly correlated systems is a notoriously difficult challenge. However, the fundamental theorems of static and also time-dependent (TD) DFT 2] are exact. Therefore in principle these systems should be accessible and the failure to describe them within (TD)DFT has to be accredited to shortcomings of the approximations for the exchange-correlation (xc) functional used in practice.

An exact property of the static xc functional is the derivative discontinuity at integer occupancy [3]. Recently the crucial importance of this property in the description of strong correlations has been recognized in, e.g., the Hubbard model [4, 5], gaps in strongly correlated molecules [6], Coulomb blockade (CB) 7] and the Mott transition [8].

For non-equilibrium situations, the development of TD $\mathrm{xc}$ functionals is still at its infancy. Typically one uses the so-called adiabatic approximation by importing functionals constructed for static DFT to the time domain. In the context of quantum transport, one of the most debated questions is whether (or under which circumstances) static DFT within the Non-Equilibrium Green's Function (NEGF) approach can yield accurate conductances. In a seminal paper, Schmitteckert and Evers [9] provided strong numerical evidence that the Kohn-Sham (KS) conductance of single channel correlated model junctions is in good agreement with the exact one. Later, the accuracy of the KS conductance has been explained and assessed using the Friedel sum rule 10. In general, however, the DFT+NEGF formalism is incomplete. A proper TDDFT treatment [1, 12] leads to dynamical xc corrections [13], the quantitative importance of which has yet to be clarified.

In this Letter we show that the derivative discontinuity is the necessary and sufficient ingredient to describe the Kondo effect 14] in the zero temperature conductance using DFT+NEGF. We propose an analytic KS potential for the Anderson model based on finite-temperature DFT [15] for an isolated impurity. For this approximate potential, the derivative discontinuity and, as a consequence also the conductance plateau, emerge naturally in the zero-temperature limit. At finite temperatures, instead, we demonstrate that the DFT+NEGF approach is not sufficient. Although our potential is exact at the particle-hole (ph) symmetric point, at the Kondo temperature the KS and the exact conductances differ by almost one order of magnitude. We thus give a first explicit example where the exact dynamical xc corrections can be quantified and show that they can be as large as the quantum of conductance.

We consider the Anderson model for a single nonmagnetic impurity attached to two non-interacting leads. The Hamiltonian of this system is given by

$$
\hat{H}=\hat{H}_{I}+\sum_{\alpha=L, R} \hat{H}_{\alpha}+\hat{H}_{T} .
$$

Here, $\hat{H}_{\alpha}=-\sum_{\sigma} \sum_{i=1}^{\infty}\left(V \hat{c}_{i+1 \alpha, \sigma}^{\dagger} \hat{c}_{i \alpha, \sigma}+\right.$ h.c. $)$ describes, in standard notation, the tight-binding lead $\alpha=L, R$ while $\hat{H}_{T}=-\sum_{\alpha, \sigma}\left(V_{\text {link }} \hat{c}_{1 \alpha, \sigma}^{\dagger} \hat{d}_{\sigma}+\right.$ h.c. $)$ accounts for the (symmetric) coupling between the impurity and the leads. In this work we focus on the half-filled system and take $V_{\text {link }} \ll V$. In this parameter range the only relevant energy scale for electron tunneling is $\gamma=2 V_{\text {link }}^{2} / V$ (wide- 
band limit). The impurity Hamiltonian is

$$
\hat{H}_{I}=v_{0} \hat{n}+U \hat{n}_{\uparrow} \hat{n}_{\downarrow}
$$

where $v_{0}$ is the on-site energy (or gate voltage), $U$ is the charging energy, $\hat{n}_{\sigma}=\hat{d}_{\sigma}^{\dagger} \hat{d}_{\sigma}$ is the number operator for electrons of spin $\sigma$ at the impurity and $\hat{n}=\hat{n}_{\uparrow}+\hat{n}_{\downarrow}$.

For a DFT treatment of the problem, the first task is to construct an approximation to the xc potential. Here we propose a KS potential based on an isolated impurity with Hamiltonian $\hat{H}_{I}$ in contact with a thermal bath at inverse temperature $\beta$ and chemical potential $\mu$ [16]. The non-interacting KS Hamiltonian then reads

$$
\hat{H}_{I}^{\mathrm{s}}=v_{\mathrm{s}} \hat{n} .
$$

For both Hamiltonians $\hat{H}_{I}$ and $\hat{H}_{I}^{\text {s }}$, the eigenstates for electron occupation zero, one, and two are, respectively, $|0\rangle,|\uparrow\rangle,|\downarrow\rangle$, and $|\uparrow \downarrow\rangle$ with eigenvalues $0, v_{0}, v_{0}$, and $2 v_{0}+U$ for $\hat{H}_{I}$ and $0, v_{\mathrm{s}}, v_{\mathrm{s}}$, and $2 v_{\mathrm{s}}$ for $\hat{H}_{I}^{\mathrm{s}}$. The density of the interacting impurity is

$$
n=\operatorname{Tr}\left\{e^{-\beta\left(\hat{H}_{I}-\mu \hat{n}\right)} \hat{n}\right\} / Z
$$

where $Z=\operatorname{Tr}\left\{e^{-\beta\left(\hat{H}_{I}-\mu \hat{n}\right)}\right\}$ is the grand-canonical partition function. Equation (44) depends only on $\tilde{v}_{0}=v_{0}-\mu$ and the function $n\left(\tilde{v}_{0}\right)$ can be inverted explicitly as

$$
\tilde{v}_{0}(n)=-U-\frac{1}{\beta} \ln \left(\frac{\delta n+\sqrt{\delta n^{2}+e^{-\beta U}\left(1-\delta n^{2}\right)}}{1-\delta n}\right),
$$

with $\delta n=n-1$. The Hartree-exchange-correlation (Hxc) part of the KS potential can then be obtained from

$$
v_{\mathrm{Hxc}}(n)=\tilde{v}_{\mathrm{s}}(n)-\tilde{v}_{0}(n)=\frac{U}{2}+g(n-1)
$$

where $g(x)=\frac{U}{2}+\frac{1}{\beta} \ln \left(\frac{x+\sqrt{x^{2}+e^{-\beta U}\left(1-x^{2}\right)}}{1+x}\right)$. This is an odd function of its argument, $g(-x)=-g(x)$, and therefore $v_{\mathrm{Hxc}}(n=1)=\frac{U}{2}$ for all temperatures. We note in passing that Eq. (6) can also be obtained by differentiation of the Hxc part of the grand canonical potential with respect to $n$. The Hxc potential (6) is shown in the left panel of Fig. 1 for different values of the temperature $T=1 / \beta$. In the limit $T \rightarrow 0$ it becomes a simple step function with a step of height $U$ at $n=1$. Thus, the $T=0$ discontinuity of the xc potential emerges naturally from our grand canonical DFT treatment.

For the Anderson Hamiltonian (1) we make a local approximation and assume that the KS potential vanishes in the leads while on the impurity is given by $v_{\mathrm{s}}(n)=v_{0}+v_{\mathrm{Hxc}}(n)$. We expect this approximation to be accurate for $U / \gamma \gg 1$. The KS Hamiltonian then reads $\hat{H}^{\mathrm{s}}=\hat{H}_{I}^{\mathrm{s}}+\hat{H}_{L}+\hat{H}_{R}+\hat{H}_{T}$ with $\hat{H}_{I}^{\mathrm{s}}$ from Eq. (3). Using standard embedding techniques the solution of the
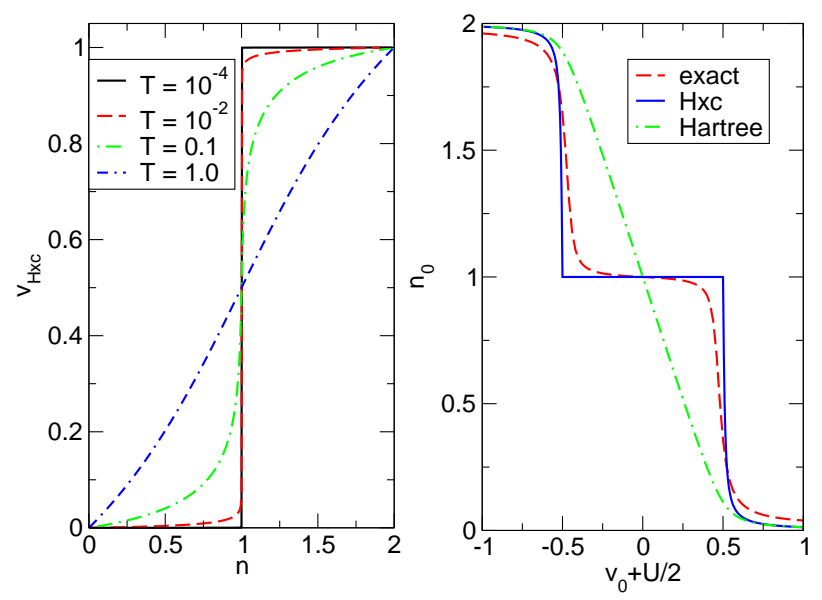

FIG. 1: Left panel: Hxc potential of Eq. (6) for different temperatures $T=1 / \beta$. Right panel: Hartree and Hxc selfconsistent impurity density in comparison to exact results for $T=0$ and $\gamma=10^{-2}$. Energies are given in units of $U$.

KS equations are reduced to the self-consistent solution of a single equation for the density $n=n_{0}$

$$
n_{0}=\frac{2}{\pi} \int_{-\infty}^{\infty} \mathrm{d} \omega \frac{\gamma}{\left(\omega-v_{\mathrm{s}}\left(n_{0}\right)\right)^{2}+\gamma^{2}} f_{\beta}(\omega)
$$

where $f_{\beta}(\omega)=1 /\left(1+e^{\beta(\omega-\mu)}\right)$ is the Fermi distribution. For low temperatures, the Hxc potential varies very rapidly for $n_{0} \sim 1$, i.e., in the regime in which we are interested (see below). Therefore, instead of solving for $n_{0}$ it is numerically much more advantageous to express $n_{0}$ in the l.h.s. of Eq. (7) in terms of $v_{\mathrm{s}}$ and solve for $v_{\mathrm{s}}$.

The resulting self-consistent solution of the density as a function of $v_{0}$ exhibits a plateau between $-U$ and 0 with value $n_{0}=1$ (see right panel of Fig. 1). This is in agreement with the exact solution of the Anderson model [17. Using the DFT+NEGF formalism we then proceed to calculate the conductance $G$ from

$$
\frac{G}{G_{0}}=-\int_{-\infty}^{\infty} \mathrm{d} \omega \mathcal{T}(\omega) \frac{\partial f_{\beta}(\omega)}{\partial \omega}
$$

where

$$
\mathcal{T}(\omega)=\frac{\gamma^{2}}{\left(\omega-v_{\mathrm{s}}\left(n_{0}\right)\right)^{2}+\gamma^{2}}
$$

is the zero-bias KS transmission function and $G_{0}=1 / \pi$ is the quantum of conductance.

In the left panel of Fig. 2 we plot $G$ as a function of $v_{0}$ using the Hxc potential of Eq. (6) for $T=10^{-2} T_{\mathrm{K}}$, with the Kondo temperature $T_{\mathrm{K}}=\sqrt{2 U \gamma} \exp \left(\frac{\pi v_{0}\left(v_{0}+U\right)}{4 \gamma U}\right)$ [18]. For comparison we also report the results within the Hartree approximation, i.e., for $v_{\mathrm{s}}=v_{0}+U n_{0} / 2$, and the accurate Numerical Renormalization Group (NRG) results from Ref. 19. The first remarkable feature is the plateau in the Hxc conductance with value $G_{0}$ in the 

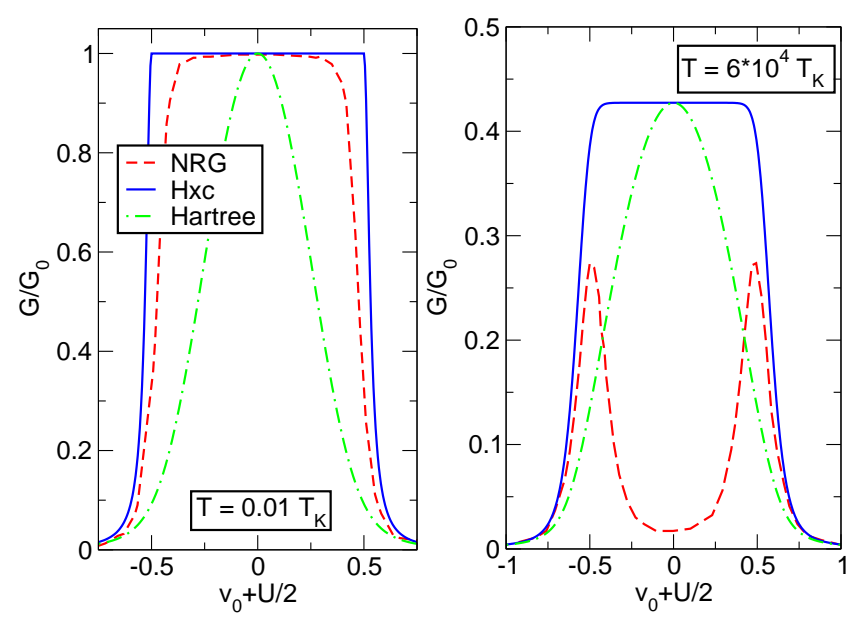

FIG. 2: Hartree, Hxc and NRG [19] conductances versus $v_{0}$ for two different temperatures. The parameters are: $\mu=0$, $\gamma=\pi \times 10^{-2}\left[T_{\mathrm{K}}=6.6 \times 10^{-7}\right]$. All energies are given in units of $U$.

range $-U \leq v_{0} \leq 0$, in good agreement with the exact result. This is a direct consequence of the discontinuity of the proposed $v_{\mathrm{Hxc}}$ at $n=1$ which guarantees that $v_{\mathrm{s}}$ is pinned to the Fermi energy. In contrast, any continuous potential, like the Hartree potential, cannot capture the plateau. Physically, the plateau cannot be explained solely in terms of $\mathrm{CB}$, according to which $G$ should be peaked at the end-points of the plateau and should instead be small at the ph symmetric point $v_{0}=-U / 2$. The value $G=G_{0}$ at $v_{0}=-U / 2$ is due to the formation of a resonant many-body singlet state between the spin of the impurity electron and the spin of the screening cloud, i.e., the Kondo effect. The first important finding of this Letter is therefore that the Kondo effect in $G(T=0)$ is within reach of the DFT+NEGF approach provided that the approximate $v_{\mathrm{Hxc}}$ has the proper discontinuity at integer $n$.

The theoretical possibility of describing the conductance in the Kondo regime within DFT+NEGF can be understood in two ways. (1) For proportional coupling the Meir-Wingreen formula [20] at $T=0$ yields

$$
\frac{G}{G_{0}}=\gamma^{2}|\mathcal{G}(\mu)|^{2} \frac{\gamma-\operatorname{Im}[\Sigma(\mu)]}{\gamma}
$$

where $\mathcal{G}^{-1}(\omega)=\left(\omega-v_{0}-\Sigma(\omega)+i \gamma\right)$ is the impurity Green's function and $\Sigma$ is the many-body self-energy. Since quasi-particles at the Fermi energy have an infinitely long life-time then $\operatorname{Im}[\Sigma(\mu)]=0$. From Eq. (10) we thus see that it is possible to reproduce the exact conductance in a KS system with $v_{\mathrm{s}}=v_{0}+\operatorname{Re}[\Sigma(\mu)]$. (2) From the Friedel sum rule we know that at $T=0$ the conductance of the Anderson model is completely determined by the density at the impurity [10], i.e., $G=G\left(n_{0}\right)$. Since exact DFT yields the exact density then it must also yield the exact conductance. Note that

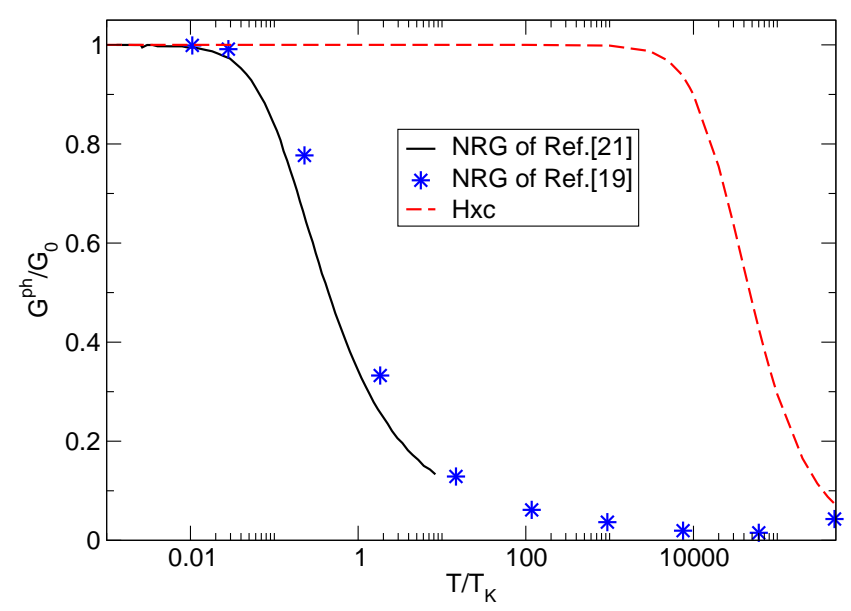

FIG. 3: NRG conductances from Ref. 19] [stars(blue)] and Ref. 21] [solid(black)] against the exact Hxc conductance [dashed(red)] at the ph symmetric point versus temperature.

the two explanations above are complementary but not equivalent since the exact $\mathrm{KS}$ potential is not rigorously zero in the neighborhood of the impurity. It is also worth stressing that the equality between the exact and KS conductances does not follow from an equality between the corresponding spectral functions. The latter are completely different, although the Kondo peak (of width $T_{\mathrm{K}}$ ) and the KS peak (of width $\gamma$ ) both occur at the Fermi energy.

At finite temperature $G$ does not depend on $n_{0}$ alone. In the right panel of Fig. 2 we display the NRG, Hxc and Hartree conductances as a function of $v_{0}$ for $T=$ $6 \cdot 10^{4} T_{\mathrm{K}}$. The failure of the Hxc and Hartree approximation in reproducing both the dip at $v_{0}=-U / 2$ as well as the CB side peaks is evident. In Fig. 3we compare the Hxc conductance with the exact conductance [19, 21] for different temperatures at the ph symmetric point. While the Kondo peak is strongly suppressed for $T \gtrsim 10 T_{\mathrm{K}}$, the DFT+NEGF approach predicts a conductance $G_{0}$ up to much higher temperatures. For $T \gtrsim U$, thermal fluctuations destroy the $\mathrm{CB}$ and the $\mathrm{KS}$ and exact conductances merge and approach zero for $T \rightarrow \infty$.

Is it possible to reproduce the temperature behavior of the exact $G$ by improving the quality of the KS potential? The answer to this question is negative since at the ph symmetric point our Hxc potential is exact. For $v_{0}=$ $-U / 2$ the Hamiltonian in Eq. (10) is invariant under the transformation $\hat{c}_{i \sigma, \alpha} \rightarrow(-1)^{i+1} \hat{c}_{i \alpha, \sigma}^{\dagger}$ and $\hat{d}_{\sigma} \rightarrow \hat{d}_{\sigma}^{\dagger}$, and hence the density is unity for all sites at all temperatures. The KS potential which reproduces this density is zero everywhere (in leads and impurity) for all $T$ and $V_{\text {link. }}$.

If one cannot reproduce the exact $G$ by using the exact $\mathrm{KS}$ potential we must conclude that the DFT+NEGF approach is not sufficient. To trace back the origin of the problem we resort to TDDFT [22] and its lattice version 23. In TDDFT it is possible to reproduce the exact 
TD longitudinal current in a system of non-interacting electrons. In Ref. 11 it was shown that if a steady-state is reached in the long-time limit then the current is given in terms of a Landauer-like formula with $\mathrm{KS}$ bias $V_{\alpha}+$ $V_{\alpha, \mathrm{xc}}$, where $V_{\alpha}$ is the physical bias and $V_{\alpha, \mathrm{xc}}$ is the xc correction. Then, to first order the current becomes (see also Ref. 13)

$$
I=G_{0}\left(V_{L}-V_{R}+V_{L, \mathrm{xc}}-V_{R, \mathrm{xc}}\right) \int d \omega \frac{\partial f_{\beta}(\omega)}{\partial \omega} \mathcal{T}(\omega) .
$$

Consequently, the finite temperature conductance $G=$ $I /\left(V_{R}-V_{L}\right)$ coincides with that of the DFT+NEGF approach in Eq. (8) only provided that $V_{\alpha, \mathrm{xc}}=0$. The exact expression for the xc bias is (to first order in $V_{\alpha}$ )

$$
V_{\alpha, \mathrm{xc}}=\lim _{i \rightarrow \infty} \sum_{r} f_{\mathrm{xc}}(i \alpha, r) \delta n_{r}
$$

where the sum runs over all sites $r$ of the model, $\delta n_{r}$ is the first-order density change in site $r$ and $f_{\mathrm{xc}}\left(r, r^{\prime}\right)$ is the zero-frequency xc kernel of TDDFT. In most commonly used local approximations $f_{\mathrm{xc}}\left(r, r^{\prime}\right) \propto \delta_{r r^{\prime}}$ and hence $V_{\alpha, \mathrm{xc}}$ vanishes since $\delta n_{r}=0$ deep inside the leads; as a result, it is often neglected altogether. The relevance of the dynamical xc correction has long been debated [9, 24]. The second important finding of this Letter is that at finite temperature the dynamical xc corrections are absolutely essential. They are not only important for the correct suppression of the Kondo peak but also for a quantitive description of the $\mathrm{CB}$ side peaks.

For the Anderson model we can find an explicit form of the dynamical xc correction to $G$ in terms of the xc kernel. The linear density change at site $r$ is

$$
\delta n_{r}=\sum_{r^{\prime} \in L} P_{r r^{\prime}} V_{L}+\sum_{r^{\prime} \in R} P_{r r^{\prime}} V_{R}+P_{r 0} \frac{U}{2} \delta n_{0}
$$

where $P$ is the zero-frequency polarization. In linear response TDDFT $P$ can be calculated from the xc kernel as $P=P_{0}+P_{0} f_{\mathrm{xc}} P, P_{0}$ being the non-interacting polarization. Substituting Eq. (13) into Eq. (12) and exploiting the symmetry of the $L$ and $R$ leads we find from Eq. (11) that the exact conductance at any temperature reads

$$
\frac{G}{G_{0}}=-\left(1+Q_{\mathrm{xc}}\right) \int d \omega \frac{\partial f_{\beta}(\omega)}{\partial \omega} \mathcal{T}(\omega) .
$$

Here $\mathcal{T}(\omega)$ is the KS transmission of Eq. (9) and

$$
Q_{\mathrm{xc}}=\lim _{i \rightarrow \infty} \sum_{r} \sum_{r^{\prime} \neq 0}(-1)^{\epsilon_{\alpha}\left(r^{\prime}\right)} f_{\mathrm{xc}}(i \alpha, r) P_{r r^{\prime}}
$$

with $\epsilon_{\alpha}\left(r^{\prime}\right)=0$ if $r^{\prime} \in \alpha$ and 1 otherwise. At $T=0$ we have $Q_{\mathrm{xc}}=0$ but for $T \gtrsim T_{\mathrm{K}}$ it must be $Q_{\mathrm{xc}} \gtrsim-1$. The quantity $1+Q_{\mathrm{xc}}$ can be interpreted as a $\mathrm{KS}$ dielectric function as it measures the ratio between the KS and the Hartree screening.
In conclusion we proposed a finite-temperature DFT scheme to construct approximate xc functionals for correlated systems weakly connected to leads. The resulting $\mathrm{KS}$ potential in the zero-temperature limit exhibits a discontinuity at integer number of particles, as it should. We showed that the discontinuity is essential to reproduce the conductance plateau due to the Kondo effect within the DFT+NEGF approach [25]. For temperatures larger than $T_{\mathrm{K}}$, however, the exact $\mathrm{KS}$ conductance overestimates the exact conductance by an order of magnitude. We traced back the origin of this problem to the lack of dynamical xc corrections which we expect to be relevant not only in this context but also in the description of finite temperature and finite bias transport experiments of weakly coupled molecular junctions.

S. K. acknowledges funding by the "Grupos Consolidados UPV/EHU del Gobierno Vasco" (IT-319-07) and the European Community's Seventh Framework Programme (FP7/2007-2013) under grant agreement No. 211956.

[1] A Primer in Density Functional Theory, Vol. 620 of Lecture Notes in Physics, edited by C. Fiolhais, F. Nogueira, and M. Marques (Springer, Berlin, 2003).

[2] Time-Dependent Density Functional Theory, Vol. 706 of Lecture Notes in Physics, edited by M.A.L. Marques, C.A. Ullrich, F. Nogueira, A. Rubio, K. Burke, and E.K.U. Gross (Springer, Berlin, 2006).

[3] J.P. Perdew, R.G. Parr, M. Levy, and J.L. Balduz, Phys. Rev. Lett. 49, 1691 (1982).

[4] O. Gunnarsson and K. Schönhammer, Phys. Rev. Lett. 56, 1968 (1986); K. Schönhammer, O. Gunnarsson, and R. M. Noack, Phys. Rev. B 52, 2504 (1995).

[5] N.A. Lima, L.N. Oliveira, and K. Capelle, Europhys. Lett. 60, 601 (2002); N.A. Lima, M.F. Silva, L.N. Oliveira, and K. Capelle, Phys. Rev. Lett. 90, 146402 (2003).

[6] P. Mori-Sanchez, A. J. Cohen, and W. T. Yang, Phys. Rev. Lett., 102, 066403 (2009).

[7] S. Kurth, G. Stefanucci, E. Khosravi, C. Verdozzi, and E.K.U. Gross, Phys. Rev. Lett. 104, 236801 (2010).

[8] D. Karlsson, A. Privitera and C. Verdozzi, Phys. Rev. Lett. 106, 116401 (2011).

[9] P. Schmitteckert and F. Evers, Phys. Rev. Lett. 100, 086401 (2008).

[10] H. Mera, K. Kaasbjerg, Y. M. Niquet, and G. Stefanucci, Phys. Rev. B 81, 035110 (2010); H. Mera and Y. M. Niquet, Phys. Rev. Lett. 105, 216408 (2010).

[11] G. Stefanucci and C.-O. Almbladh, Phys. Rev. B 69, 195318 (2004); Europhys. Lett. 67, 14 (2004).

[12] F. Evers, F. Weigend, and M. Koentopp, Phys. Rev. B 69, $235411(2004)$.

[13] M. Koentopp, K. Burke, and F. Evers, Phys. Rev. B 73, 121403 (2006); G. Stefanucci, S. Kurth, A. Rubio, and E.K.U. Gross, in Molecular and Nano Electronics: Analysis, Design, and Simulation, 17, edited by J. Seminario (Elsevier, Amsterdam, 2006).

[14] J. Kondo, Prog. of Theor. Phys. 32, 37 (1964).

[15] N. Mermin, Phys. Rev. 137, A1441 (1965). 
[16] After submission of our manuscript, F. Evers and P. Schmitteckert, arXiv:1106.3658 (2011) presented a study on the same model.

[17] P. B. Wiegmann and A. M. Tsvelick, J. Phys. C 16, 2281 (1983).

[18] I. L. Aleiner, P. W. Brouwer, and L. I. Glazman, Phys. Rep. 358, 309 (2002).

[19] W. Izumida and O. Sakai, J. Phys. Soc. Jpn. 74, 103 (2005).

[20] Y. Meir and N.S. Wingreen, Phys. Rev. Lett. 68, 2512 (1992).

[21] T. A. Costi, Phys. Rev. Lett. 85, 1504 (2000).

[22] E. Runge and E.K.U. Gross, Phys. Rev. Lett. 52, 997 (1984).

[23] R. Baer, J. Chem. Phys. 128, 044103 (2008); C. Verdozzi,
Phys. Rev. Lett. 101, 166401 (2008); Y. Li and C. A. Ullrich, J. Chem. Phys. 129, 044105 (2008); G. Stefanucci, E. Perfetto, and M. Cini, Phys. Rev. B 81, 115446 (2010); I. V. Tokatly, Phys. Rev. B, 035127 (2011); S. Kurth and G. Stefanucci, Chem. Phys. , in press (2011); doi:10.1016/j.chemphys.2011.01.016.

[24] N. Sai, M. Zwolack, G. Vignale, and M. Di Ventra, Phys. Rev. Lett. 94, 186810 (2005); J. Jung, P. Bokes, and R.W. Godby, Phys. Rev. Lett. 98, 259701 (2007); G. Vignale and M. Di Ventra, Phys. Rev. B 79, 014201 (2009).

[25] The same conclusion was concomitantly reached by J.P Bergfield, Z.-F. Liu, K. Burke, and C.A. Stafford, arXiv:1106.3104 (2011), and by P. Tröster, P. Schmitteckert, and F. Evers, arXiv:1106.3669 (2011). 